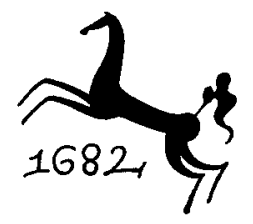

Germanistische Abhandlungen

JeAn Pauls »Komet « 
This page intentionally left blank 
UWE SCHWEIKERT

\title{
Jean Pauls »Komet»
}

\author{
SELBSTPARODIE
}

DER KUNST

$\longrightarrow$

J. B. METZLERSCHE

VERLAGSBUCHHANDLUNG

STUTTGART 
Germanistische Abhandiungen 35

Die in [ ] erscheinenden Ziffern verweisen auf die Anmerkungen

ISBN 978-3-476-ooi 88-7

ISBN 978-3-476-02984-3 (eBook)

DOI $10.1007 / 978-3-476-02984-3$

Die vorliegende Arbeit wurde 1969 von der Philosophischen Fakultät II der LudwigMaximilians-Universität zu München unter dem Titel SStudien zu Jean Pauls Komet. Der Komet als ironisch-selbstparodistisches Schlußwerk Jean Pauls als Dissertation angenommen.

(C) 197 I Springer-Verlag GmbH Deutschland Ursprünglich erschienen bei J.B. Metzlersche Verlagsbuchhandlung und Carl Ernst Poeschel Verlag GmbH in Stuttgart 1971 
Kunst ist Wahrheit -

die Wahrheit über den Künstler

(Thomas Mann, Leiden und Größe

Richard Wagners) 
Meinen Eltern 


\section{INHALT}

VORWORT

IX

I. VorÜBERLEgUNGen (Zur ForsChUNGSLAGe SOWIe ZUR ENTSTEHUNGSGESCHICHTE DES $\gg$ KOMET«) . . . . . . . . . . . . . . .

II. Jean Pauls Spätwerk im Rahmen des Gesamtwerks (Zur ProBLEMSTELLUNG DER ARBEIT).

III. MARGGRAF UND DER ICH-WAHN . . . . . . . . . . . . . I8

x. Apotheker und Fürst . . . . . . . . . . . . . . . I8

2. »Lebens-Libertin von innen« . . . . . . . . . . . . . 20

3. Nikolaus' "Selber-Unheiligsprechung « . . . . . . . . . . 23

4. Luft- und Atherschlösser . . . . . . . . . . . . . . . . . 27

5. Größenlehre der Phantasie . . . . . . . . . . . . . . 34

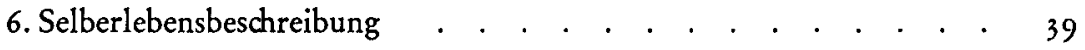

IV. Amanda oder die Puppe als Geliebte . . . . . . . . . . 44

I. Das Fernrohr . . . . . . . . . . . . . . . . . . 44

2. Seelenbräutigam der Puppen . . . . . . . . . . . . . 46

3. Ein Vorausverwundeter . . . . . . . . . . . . . . . ${ }_{4} 6$

4. Die Kunstfigur als Lebenssymbol . . . . . . . . . . . . 48

s. Nikolaus' Pygmalions-Schicksal . . . . . . . . . . . . . so

6. Abbild und Urbild . . . . . . . . . . . . . . . . . 52

7. Amanda als Geliebte . . . . . . . . . . . . . . . . $\$ 4$

8. Die poetische Liebe . . . . . . . . . . . . . . . 58

9. Die zu Liebende . . . . . . . . . . . . . . . . . 60

V. Der Ledermensch . . . . . . . . . . . . . . . . . 62

r. Ein Auftritt aus dem Nichts . . . . . . . . . . . . . . $\mathbf{6 2}_{2}$

2. Zur Genesis der Gestalt . . . . . . . . . . . . . . . 63

3. Kains Schlußrede. . . . . . . . . . . . . . . . . 65

4. Kain und Christus . . . . . . . . . . . . . . . . 66

5. Nikolaus und Kain . . . . . . . . . . . . . . . . . 68

6. Leibgeber-Schoppe-Roquairol-Kain . . . . . . . . . . . 70

7. Die Vernichtung der Kunst durch Kunst . . . . . . . . . 74

8. Vollendung im Fragment . . . . . . . . . . . 76 
VI. Der Dichter als Wetterprophet . . . . . . . . . . . . 78

r. Der Auftritt des Kandidaten Richter . . . . . . . . . . $7_{78}^{8}$

2. Der Kandidat als Figur . . . . . . . . . . . . . . . 79

3. Ich-Projektion . . . . . . . . . . . . . . . . . . $8 \mathrm{I}$

Vil. Die Hofnarren . . . . . . . . . . . . . . . . . . 83

I. Süptitz: Der philosophische Systemzwang . . . . . . . . . 84

2. Renovanz: Der sadistische Realismus . . . . . . . . . 88

3. Worble: Die Ernüchterung des Humoristen . . . . . . . . 90

4. Raphael: Kunst als Halluzination . . . . . . . . . . . . $9^{6}$

VIII. DeR Komet und die Romantik . . . . . . . . . . . . . IOz

IX. Prolegomena zu einer Bestimmung von Jean Pauls Spätstil . . i i I

x. DeR KOMET: >Fabula< oder >Historia<? Zur poetologischen Selbstreflexion des Dichters. . . . . . . . . . . . . . . . . III

2. Stilistische Besonderheiten . . . . . . . . . . . . . . II7

X. Kunst und Gesellschaft im $»$ Komet« . . . . . . . . . . ${ }^{2} 24$

I. Der satirische Gesellschaftsroman . . . . . . . . . . . . I24

2. Der Komet als Selbstparodie des Dichters. . . . . . . . . r $3 \mathbf{I}$

ANMERKUNGen . . . . . . . . . . . . . . . . . . . . . . . .

LITERATURVERZEICHNIS . . . . . . . . . . . . . . . . . . ${ }^{164}$ 


\section{VORWORT}

Allenthalben in der neueren Sekundärliteratur wird Jean Pauls Spätwerk sironische genannt $[\mathrm{I}]$, ohne daß dabei eine genaue und eingehende Untersuchung des so bezeichneten Gegenstands stattfände. Die Klassifizierung dient nur zur Abgrenzung von Hauptwerk und Spätwerk; dieses selbst bleibt außerhalb der literarwissenschaftlichen Bemühungen. Hier nun will die vorliegende Arbeit - in deren Mittelpunkt Jean Pauls letzter Roman Der Komet steht - Abhilfe schaffen.

$\mathrm{Zu}$ begründen bleibt, warum der Verfasser trotzdem von einer theoretischen Klärung der Begriffe Ironie, Parodie, Humor etc. Abstand genommen hat. Die Untersuchung begäbe sich damit auf ein Feld, das zwar wichtig, aber fast uferlos ist. Gesicherte literarwissenschaftliche Definitionen dieser Begriffe nämlich gibt es nicht. Das gilt insbesondere für die Ironie: "Die Verwendung des Begriffes Ironie in literarischen Zusammenhängen ist nicht eindeutig festgelegt " (Beda Allemann) [2]. Und Helmut Arntzen spricht von den win sich problematischen Begriffen des Humors und der Ironie" [3]. Im allgemeinen werden sie in einem mehr oder weniger metaphorischen Sinn angewandt. Sie wären für jeden Autor, ja beinahe für jedes Werk neu zu bestimmen. So weit das in unserem Fall möglich und auch notwendig ist, wurde es versucht.

Dazu kommt bei Jean Paul eine weitere Schwierigkeit. Alle die genannten Begriffe stehen im Zentrum seiner Vorschule der Asthetik. Die bisherige Forschung nun neigte dazu, eine Ubereinstimmung von ästhetischer Theorie und dichterischem Werk als gegeben vorauszusetzen; sie interpretierte - wenn auch nicht durchweg - das Werk anhand der Theorie. Diese Praxis aber muß man in Frage stellen [4]. Solange die Vorschule nicht auf ihre ,normative, Verbindlichkeit für Jean Paul selbst sowie auf ihren Zusammenhang bzw. Unterschied zur zeitgenössischen Kunsttheorie der Klassik und Romantik hin untersucht ist [ 5$]$, hat eine Anwendung ihrer theoretischen Postulate auf das Werk nur wenig Sinn. Man hat allzu schnell von einem Standpunkt aus geurteilt, von dem selbst erst zu beweisen wäre, daß er der richtige ist. Für das Spätwerk zumindest ist seine Gültigkeit entschieden anzuzweifeln.

Aus diesem Grunde geht der Verfasser in erster Linie von den Fakten, den Werken aus. Er macht sich dabei jene Goethesche Maxime zu eigen, der zufolge das Faktische die Theorie schon mit enthält: "Das Höchste wäre, zu begreifen, daß alles Faktische schon Theorie ist " (Wilhelm Meisters Wanderjabre - Betrachtungen im Sinne der Wanderer).

Im Komischen Anhang zum Titan kommt Jean Paul darauf zu sprechen, wie schwierig es sei, die komplexe Einheit eines großen Kunstwerks - wie Mozarts 
Zauberflöte es sei - auf einmal zu fassen: "Muß hier nicht r) das genialische Durcheinanderarbeiten aller Instrumente, dann das ebenso große aller Schauspieler, 2) ihre optische und 3) akustische Mimik, dann 4) das poetische Stück selber und endlich 5) das Gepränge der Verzierung auf einmal gefasset und genossen werden?" (III 900f). Hinter dieser Einsicht verbirgt sich eine versteckte Selbstcharakteristik; denn sinngemäß gilt das auch für seine eigenen Romane. Die wissenschaftliche Unteruchung aber geht - scheinbar - einen entgegengesetzten Weg: sie zergliedert, was eine Einheit darstellt. Sie fächert das Kunstwerk in seine einzelnen Aspekte auf. Diese aber sind nicht nur sukzessive, sondern auch in ihrem Zuammenhang zu sehen und zu lesen. Denn alle hier behandelten Aspekte des Komet führen zu seinem zentralen Motiv - dem der Selbstparodie - hin.

Die Untersuchung folgt zwar zuallererst einer wissenschaftlichen Zielsetzung. Trotzdem können ihr Gegenstand und ihre Ergebnisse mehr als nur >akademisches Interesse beanspruchen. So wenigstens hoft es der Verfasser. Daß er im übrigen eher den freundschaftlichen als den feindseligen Auslegern - welche Unterscheidung Jean Paul selbst einmal vornahm - sich zurechnet, will er nicht verbergen. »Unter einem freundschaflichen Ausleger « - schreibt Jean Paul - "mein' ich den, welcher in einem fremden Buche seine eigne Meinung, obwohl tief vergraben, entdeckt und mit seiner Wünschelrute erhebt « (V I I44). Die Philologie vermag Sympathie für den Gegenstand und kritische Objektivität durchaus in sich zu vereinen.

Schließlich bleibt noch ein grundlegender Mangel der Arbeit einzugestehen: sie spiegelt, auch im Negativen, den Prozeß ihrer Entstehung wider, zerfällt sie doch - zumindest streckenweise - in zwei einander widerstrebende Teile: in eine fast ausschließlich werkimmanente Interpretation des Textes sowie in die Frage nach dem historisch-gesellschaftlichen Stellenwert, den Der Komet einnimmt. Es ist das ein Mangel, der durch Retuschen allein nicht zu beheben war. Insofern dokumentiert das Buch hinsichtlich der Methode einen Stand, den der Verfasser inzwischen überwunden hat. Die Erkenntnisse und Einsichten, die es erbringt, sind damit nicht hinfällig, doch könnten sie - von heute aus geurteilt - noch wesentlich weiter getrieben und konsequenter ausgewertet werden. Die Arbeit wurde im Winter I 968 abgeschlossen; von der seither erschienenen Literatur wurde nur diejenige nachgetragen, die direkt mit dem Komet sich befaßt.

Mein Dank gilt all denen, die das Zustandekommen dieser Arbeit durch Diskussion und kritische Anregung gefördert haben, besonders Gabriele Hartmann für ihre Hilfe bei der Überarbeitung zur Drucklegung. Weiter danke ich Herrn Professor Dr. Eduard Berend, dem Leiter des Jean-Paul-Archivs in Marbach, sowie seinem Mitarbeiter Winfried Feifel für ihre vielfältige Unterstützung während meines Marbacher Aufenthaltes; vor allem jedoch meinem Lehrer Professor Dr. Werner Vordtriede, der die Arbeit angeregt und betreut hat.

Zum Schluß möchte ich darauf hinweisen, daß ich die folgenden Bücher nach den im Jean-Paul-Archiv befindlichen Handexemplaren Eduard Berends zitiert habe: Karl Freye, Jean Pauls Flegeljabre; Richard Otto Spazier, Jean Paul Friedrich Richter. Ein biographischer Commentar zu dessen Werken; Jean Pauls Nach- 
laß; Wahrheit aus Jean Pauls Leben; F. J.Schneider, Jean Pauls Altersdichtung. E. Berend hat die in diesen Büchern enthaltenen Auszüge aus unveröffentlichten Manuskripten Jean Pauls mit den Handschriften verglichen und korrigiert. Daraus erklären sich die vorkommenden Abweichungen vom gedruckten Wortlaut.

September I 970 U.S. 